\title{
Determining the adenoma detection rate and adenomas per colonoscopy by photography alone: proof-of-concept study
}

Authors

Institution
Douglas K. Rex, Kyle Hardacker, Margaret MacPhail, Farrah Rahmani, Krishna C. Vemulapalli, Charles J. Kahi

Department of Medicine, Division of Gastroenterology and Hepatology, Indiana University School of Medicine, Indianapolis, Indiana, United States submitted 30. May 2014 accepted after revision 19. November 2014

\section{Bibliography}

Dol http://dx.doi.org/ 10.1055/s-0034-1391330

Published online: 15.1.2015

Endoscopy 2015; 47: 245-250

(C) Georg Thieme Verlag KG

Stuttgart · New York

ISSN 0013-726X

\section{Corresponding author}

\section{Douglas K. Rex, MD}

Department of Medicine Division of Gastroenterology and Hepatology

Indiana University Hospital \#4100

550 N. University Boulevard Indianapolis

IN 46202

United States

Fax: +1-317-944-5449

drex@iupui.edu
Background and study aims: The adenoma detection rate (ADR) and adenomas detected per colonoscopy (APC) are measures of the quality of mucosal inspection during colonoscopy. In a resect and discard policy, pathologic assessment for calculation of ADR and APC would not be available. The aim of this study was to determine whether ADR and APC calculation based on photography alone is adequate compared with the pathologybased gold standard.

Patients and methods: A prospective, observational, proof-of-concept study was performed in an academic endoscopy unit. High definition photographs of consecutive polyps were taken, and pathology was estimated by the colonoscopist. Among 121 consecutive patients aged $\geq 50$ years who underwent colonoscopy, 268 polyps were removed from 97 patients. Photographs of conse-

\section{Introduction \\ $\nabla$}

The adenoma detection rate (ADR) is the primary measure of the quality of mucosal inspection during colonoscopy [1,2]. Higher ADRs predict better protection against colorectal cancer $[3,4]$. In addition to the ADR, there is interest in the measurement of adenomas detected per colonoscopy (APC) as a quality measure [2,5]. The ADR and APC correlate in some but not all centers [6]. Adequate ADR with low APC may reflect a "one-anddone" manipulation of ADR, so that in some settings APC or a similar measure may be a better reflection of the quality of mucosal inspection than ADR [2,5 - 7].

Technologies including narrow-band imaging (NBI; Olympus America Corp., Center Valley, Pennsylvania, USA), confocal laser microscopy, endocytoscopy, autofluorescence, the Fujinon Intelligent Chromoendoscopy system (Fujifilm Medical Systems, Wayne, New Jersey, USA), and the Pentax i-scan (Pentax Medical, Montvale, New Jersey, USA) have been shown to allow accu- cutive polyps were reviewed by a second endoscopist.

Results: The resect and discard policy applied to lesions that were $\leq 5 \mathrm{~mm}$ in size. When only photographs of lesions that were ultimately proven to be adenomas were included, the reviewer assessed ADR and APC to be lower than that determined by pathology (absolute reductions of 6.6\% and 0.17 , and relative reductions of $12.6 \%$ and $13.1 \%$ in ADR and APC, respectively). When all photographs were included for calculation of ADR and APC, the reviewer determined the ADR to be $3.3 \%$ lower (absolute reduction) and the APC to be the same as the rates determined by pathology.

Conclusions: In a simulated resect and discard strategy, a high-level detector can document adequate ADR and APC by photography alone.

rate estimation of pathology in colorectal polyps in real time $[8,9]$. Two practical roles for these technologies have been proposed $[9,10]$. The first role is within the policy of "resect and discard," in which diminutive or small polyps, for which the pathology is predicted by endoscopy with high confidence, are resected and then discarded without pathologic assessment [9-12]. Modeling indicates that the resect and discard policy is cost effective $[13,14]$. A second proposed role is to identify diminutive rectosigmoid polyps as hyperplastic and then leave them in place, thereby avoiding the costs of both polypectomy and pathologic assessment [9]. This paradigm was proposed by the American Society for Gastrointestial Endoscopy (ASGE) [9], and endorsed by the European Society for Gastrointestinal Endoscopy [10].

Given that measurement of ADR and perhaps APC (in the future) are important to high quality colonoscopy, the introduction of resect and discard could create a problem for quality measurements, as some diminutive polyps would not be available 
for pathologic assessment and documentation of adenomas. The ASGE addressed this issue in its Preservation and Incorporation of Valuable Endoscopic Innovations document on real-time pathology assessment of colorectal polyps [9]. The ASGE indicated that the use of imaging technologies for real-time assessment must be accompanied by stored images that are of sufficient quality to create a permanent record of the basis on which the endoscopist's real-time decision was made [9]. Thus, high quality, permanently stored photographs or video recordings are the likely solution to the lack of pathology data in a resect and discard program.

Stored images of polyps are also one solution to avoiding the excess pathology costs associated with APC measurement. For example, if three separate adenomas are seen and removed from the same section of the colon, then a photograph of each polyp would prove their multiplicity for APC measurement. The three polyps could then be placed in the same bottle for pathology assessment, or potentially discarded in a resect and discard program.

To our knowledge, no previous study of using endoscopic imaging has included demonstration of adequate ADR and APC as its primary focus. In a resect and discard practice, endoscopists would refer polyp photographs for review by other expert endoscopists to prove that their ADR and APC met recommended thresholds for detection. The aim of the current study was to assess whether a known high level detector with experience in real-time diagnosis could use photography in consecutive examinations to document adequate ADR and APC.

\section{Methods}

$\nabla$

\section{Design and patients}

This prospective assessment of photography and its adequacy for documentation of ADR and APC was approved by the Indiana University Institutional Review Board.

A single colonoscopist (D.K.R.) with a proven high ADR (47\%$58 \%$ in two screening colonoscopy studies) $[11,15,16]$ acquired endoscopic images of all resected polyps from 121 consecutive colonoscopies during a 6-week period. The colonoscopist also had proven high accuracy (89\% - 94\% overall accuracy and 94\%$98 \%$ for high confidence readings in two studies) in real-time estimation of colorectal polyp histology using NBI [11,17].

Patients were included if they were aged 50 years or over. Patients were excluded if they had inflammatory bowel disease, previous colonic resection, a known polyposis syndrome, or were referred for resection of a large polyp identified by another endoscopist.

\section{Image assessment}

All polyp images were acquired using NBI and 180 series colonoscopes (Olympus America Corp.). For each polyp, the colonoscopist froze an image until a satisfactory image for capture and storage was obtained. Electronic magnification was used at the discretion of the colonoscopist. The colonoscopist estimated polyp pathology in real time using the Narrow band imaging International Colorectal Endoscopic classification (NICE) criteria [18]. Confidence in interpretation was assigned as high or low in real time. Polyp size was estimated by the colonoscopist using the longest apparent dimension of the polyp.In some cases, the polyp was sized using open forceps. The size estimated by the colonoscopist was used to assign polyps to size groups in order to assess the accuracy of the colonoscopist and the reviewer. Polyps were resected using standard techniques and sent for pathologic assessment.

\section{Calculation of ADR and APC}

ADR was the proportion of patients having one or more adenomas in the colon. APC was the total number of adenomas in all study colonoscopies divided by the total number of study colonoscopies.

ADR and APC were calculated according to: the determination made by the pathologist; the estimation of pathology made in real time during colonoscopy; and the review of stored photographs by the second endoscopist (reviewer) using NICE criteria [18].

The second endoscopist was an experienced colonoscopist who had authored a number of clinical studies of colonoscopy, had completed a training program in NBI [19], and reported using NBI in clinical practice in hundreds of cases with feedback from routine pathology reports. However, the second endoscopist's accuracy in optical biopsy had not been proven in a previous published clinical trial. No clinical information was provided to the reviewer. Thus, the reviewer was unaware of the number of polyps removed from each patient, the location of polyps in the colon, or their size or histology as estimated by the primary colonoscopist. High definition images in TIF format were displayed on a high definition monitor for the review. Each pathology estimation was also characterized as a high or low confidence interpretation. No photographs were considered by the reviewer to be of insufficient quality to make a pathology estimation.

The ADR and APC were calculated first by counting lesions verified by pathology as adenomas and then by using all adenoma guesses from the colonoscopist and the reviewer irrespective of pathology verification. It was assumed that polyps in the size categories of $6-9 \mathrm{~mm}$ and $\geq 10 \mathrm{~mm}$ would be sent for pathology (and the pathology report would be used to determine the contribution to ADR and APC), while smaller lesions ( $\leq 5 \mathrm{~mm}$ ) would be interpreted by the colonoscopist and the reviewer. Results are presented with the ADR and APC calculated when only the high confidence interpretations of small polyps by the colonoscopist were counted toward ADR and APC, with histology being used to identify adenomas among small polyps interpreted with low confidence by the colonoscopist. Results are also presented with the colonoscopist's estimations of pathology used for all small polyps regardless of the confidence rating, as some endoscopists might interpret all polyps of small size with high confidence.

\section{Statistical analysis}

The sample size calculation was performed using Stata version 13 (StataCorp, College Station, Texas, USA). Assuming a prevalence of adenomas of $50 \%, 97$ patients were needed to determine ADRs within a $95 \%$ confidence range of \pm 10 percentage points at $80 \%$ power. The agreement between the endoscopist and the reviewer was measured using Cohen's kappa. For this analysis, the 234 polyps for which the colonoscopist's estimate of pathology matched the gold standard of pathology were used. Assuming that the agreement between the colonoscopist and the reviewer would be moderate ( 0.5$)$, a sample size of 234 polyps provided a $95 \%$ confidence interval of 0.1 around the measured kappa value. The $95 \%$ confidence intervals for the ADR were calculated using the equation

$$
p \pm 1.96 \sqrt{[}[p(1-p) / \mathrm{n}]
$$


where $p$ is the ADR point estimate and $n$ is the number of procedures.

The 95\% confidence intervals for APC were calculated using the formula

$$
x \pm 1.96(\mathrm{SD} / \sqrt{\mathrm{n}})
$$

where $x$ is the APC point estimate and SD is the standard deviation of the number of adenomas detected per colonoscopy [20]. The proportion of readings with high confidence and the accuracy were compared between the colonoscopist and the reviewer using a two-sided chi-squared test. Statistical significance was set at 0.05.ADR, APC calculations, chi-squared tests, and Cohen's kappa measurement were performed using SPSS version 20 (IBM Inc., Armonk, New York, USA).

\section{Results}

\section{$\nabla$}

A total of 121 consecutive patients aged $\geq 50$ years met the eligibility criteria and were included in the study. The mean age was 64.7 years, and there were 67 women (55.4\%). The indications were polyp surveillance in 79 (65.3\%), screening in 28 (23\%), and diagnostic in 14 (11.6\%). There were 97 patients with at least one polyp removed, and 63 with at least one conventional adenoma (not including patients with only sessile serrated polyp/adenoma [SSA/P]). A total of 268 polyps or flat lesions were removed from the 97 patients, and the histology of the lesions as determined by the pathologist is shown in Table 1 . In addition, one cancer was found in an overt mass (recognized as cancer by the endoscopist and the reviewer).

- Table 2 shows the accuracy of the colonoscopist and reviewing endoscopist assessments compared with pathology on a perpolyp basis. The colonoscopist interpreted a smaller percentage of all lesions with high confidence compared with the reviewer ( $88 \%$ vs. $94 \% ; P=0.109$ ). For all lesions (regardless of size) read with high confidence by both the colonoscopist and the reviewer, the colonoscopist had higher accuracy than the reviewer (92\% vs. $83 \% ; P<0.001)$. This difference was driven largely by higher accuracy for lesions of $\leq 5 \mathrm{~mm}$ in size ( $89 \%$ vs. $80 \%$; $P<0.001$ ). For lesions interpreted with high confidence by the colonoscopist, the kappa value for the agreement between the colonoscopist and the reviewer was 0.619 for lesions $1-5 \mathrm{~mm}$ in size, 0.617 for lesions $6-9 \mathrm{~mm}$ in size, and 0.9 for lesions $\geq 10 \mathrm{~mm}$ in size.

Using the histology determined by the pathologist as the gold standard, the ADR for the 121 patients was $52.1 \%$ and the APC was 1.30 ( Table $3, \bullet$ Table 4 ).

- Table 3 shows the ADRs and APCs determined by the colonoscopist and the reviewer, according to the confidence readings and including only those adenomas that were verified by the pathologist. Table 4 shows the same data but includes all lesions called adenomas by the colonoscopist and reviewer without reference to the pathologist interpretation (i.e. some of the endoscopist and reviewer adenoma readings would be false-positive results). Table 3 and $\bullet$ Table 4 show the ADR and APC if the resect and discard policy was expanded to polyps in the 6-9-mm size range [12].

When only pathologist-verified adenomas are counted toward ADR and APC ( Table 3 ), the calculated ADR and APC rates were all numerically less than the pathologically determined ADR and APC. In the United States, the use of photography would be for lesions $\leq 5 \mathrm{~mm}$ in size evaluated with high confidence. In this situation, the reviewer established an ADR for the colonoscopist of $45.4 \%$ and an APC of 1.13 . These are absolute reductions of $6.6 \%$ and 0.17 , and relative reductions of $12.6 \%$ and $13.1 \%$ in ADR and

Table 1 Pathology findings by lesion size ( $\mathrm{n}=268$ lesions).

\begin{tabular}{|c|c|c|c|}
\hline Lesion size, mm & Adenoma, $\mathrm{n}(\%)^{1}$ & Hyperplastic and other serrated, n (\%) ${ }^{1}$ & Other $^{2}, \mathrm{n}(\%)^{1}$ \\
\hline$\leq 5$ & 109 (59.2) & $54(29.3)$ & $21(11.4)$ \\
\hline $6-9$ & $36(58.1)$ & $26(41.9)$ & $0(0)$ \\
\hline$\geq 10$ & $12(54.5)$ & $8(36.4)$ & $2(9.1)$ \\
\hline Total & $157(58.6)$ & $88(32.8)$ & $23(8.6)$ \\
\hline
\end{tabular}

1 Percentages calculated as proportion of lesion size group.

${ }^{2}$ Other consists of 13 normal tissue, 1 inflammatory, 9 not retrieved or fecal matter only recovered.

Table 2 Accuracy of interpretations by the colonoscopist and the reviewer compared with pathology-all polyps.

\begin{tabular}{|c|c|c|c|c|}
\hline & \multicolumn{2}{|c|}{ High and low confidence readings } & \multicolumn{2}{|c|}{ High confidence readings only } \\
\hline & $\begin{array}{l}\text { Adenomas interpreted as } \\
\text { adenomas, } \mathbf{n}(\%)[95 \% \mathrm{Cl}]\end{array}$ & $\begin{array}{l}\text { Nonadenomas interpreted as } \\
\text { nonadenomas, } \%[95 \% \mathrm{Cl}]\end{array}$ & $\begin{array}{l}\text { Adenomas interpreted as } \\
\text { adenomas, } \%[95 \% \mathrm{Cl}]\end{array}$ & $\begin{array}{l}\text { Nonadenomas interpreted as } \\
\text { nonadenomas, } \%[95 \% \mathrm{Cl}]\end{array}$ \\
\hline \multicolumn{5}{|l|}{ All lesions } \\
\hline Colonoscopist & $150(95.5)[92.3-98.8]$ & $81(79.4)[71.6-87.3]$ & $134(96.4)$ [93.3 - 99.5] & $75(83.3)[75.6-91]$ \\
\hline Reviewer & $132(84.1)[78.4-89.8]$ & $74(72.5)[63.9-81.2]$ & $127(85.8)[80.2-91.4]$ & $71(74.7)[66-83.5]$ \\
\hline \multicolumn{5}{|l|}{ Lesions $\geq 10 \mathrm{~mm}$} \\
\hline Colonoscopist & $11(91.7)[76-100]$ & $9(100)[N / A]$ & $10(90.9)[73.9-100]$ & $9(100)[N / A]$ \\
\hline Reviewer & $11(91.7)$ [76-100] & 8 (88.9) [68.4-100] & $11(100)[\mathrm{N} / \mathrm{A}]$ & 8 (88.9) [68.4-100] \\
\hline \multicolumn{5}{|l|}{ Lesions 6-9 mm } \\
\hline Colonoscopist & $33(91.7)$ [82.6 - 100] & $26(100)[N / A]$ & $32(94.1)[86.2-100]$ & $25(100)[N / A]$ \\
\hline Reviewer & $34(94.4)[87-100]$ & $19(73.1)[56-90.1]$ & $33(97.1)[91.4-100]$ & $18(78.3)[61.4-95.1]$ \\
\hline \multicolumn{5}{|l|}{ Lesions $\leq 5 \mathrm{~mm}$} \\
\hline Colonoscopist & $106(97.2)[94.2-100]$ & $46(68.7)[57.6-79.8]$ & 92 (97.9) [95 - 100] & $41(73.2)[61.6-84.8]$ \\
\hline Reviewer & $87(79.8)[72.3-87.4]$ & $47(70.1)[59.2-81.1]$ & $83(80.6)[72.9-88.2]$ & $45(71.4)[60.3-82.6]$ \\
\hline
\end{tabular}

N/A, not applicable. 


\begin{tabular}{|c|c|c|}
\hline & ADR, \% [95\%Cl] & $\operatorname{APC}[95 \% \mathrm{Cl}]$ \\
\hline Determined by pathology & $52.1[43.2-61]$ & $1.30[0.96-1.64]$ \\
\hline \multicolumn{3}{|l|}{$\begin{array}{l}\text { Determined by using endoscopy estimates for high confidence readings of } \\
\text { polyps } \leq 5 \mathrm{~mm} \text { in size, pathology for polyps } \geq 6 \mathrm{~mm} \text { in size or for polyps } \leq 5 \mathrm{~mm} \\
\text { in size with low confidence }\end{array}$} \\
\hline Colonoscopist & $51.2[42.3-60.1]$ & $1.28[0.8-1.46]$ \\
\hline Reviewer & $45.5[36.6-54.4]$ & $1.13[0.8-1.46]$ \\
\hline \multicolumn{3}{|l|}{$\begin{array}{l}\text { Determined by using endoscopy estimates for both high and low confidence } \\
\text { readings of polyps } \leq 5 \mathrm{~mm} \text { and pathology for polyps } \geq 6 \mathrm{~mm}\end{array}$} \\
\hline Colonoscopist & $51.2[42.3-60.1]$ & $1.27[0.93-1.61]$ \\
\hline Reviewer & $44.6[35.7-53.5]$ & $1.12[0.79-1.44]$ \\
\hline \multicolumn{3}{|l|}{$\begin{array}{l}\text { Determined by using endoscopy estimates for high confidence readings of } \\
\text { polyps }<10 \mathrm{~mm} \text { in size, and pathology for polyps } \geq 10 \mathrm{~mm} \text { in size or for polyps } \\
<10 \mathrm{~mm} \text { in size with low confidence }\end{array}$} \\
\hline Colonoscopist & $51.2[42.3-60.1]$ & $1.26[0.92-1.6]$ \\
\hline Reviewer & $45.5[36.6-54.4]$ & $1.12[0.8-1.45]$ \\
\hline \multicolumn{3}{|l|}{$\begin{array}{l}\text { Determined by using endoscopy estimates for both high and low confidence } \\
\text { readings of polyps }<10 \mathrm{~mm} \text { in size, pathology for polyps } \geq 10 \mathrm{~mm} \text { in size }\end{array}$} \\
\hline Colonoscopist & $50.4[41.5-59.3]$ & $1.25[0.91-1.59]$ \\
\hline Reviewer & $44.6[35.7-53.5]$ & $1.1[0.77-1.42]$ \\
\hline
\end{tabular}

Table 3 Adenoma detection rate and adenomas per colonoscopy pathologically confirmed adenomas only.

ADR, adenoma detection rate; APC, adenomas per colonoscopy.

\begin{tabular}{|c|c|c|}
\hline & ADR, \% [95\%Cl] & $\operatorname{APC}[95 \% \mathrm{Cl}]$ \\
\hline Determined by pathology & $52.1[43.2-61]$ & $1.30[0.96-1.64]$ \\
\hline \multicolumn{3}{|l|}{$\begin{array}{l}\text { Determined by using endoscopy estimates for high confidence readings of } \\
\text { polyps } \leq 5 \mathrm{~mm} \text { in size, pathology for polyps } \geq 6 \mathrm{~mm} \text { in size or for polyps } \leq 5 \mathrm{~mm} \\
\text { in size with low confidence }\end{array}$} \\
\hline Colonoscopist & $55.4[46.5-64.3]$ & $1.43[1.07-1.79]$ \\
\hline Reviewer & $49.6[40.7-58.5]$ & $1.31[0.96-1.67]$ \\
\hline \multicolumn{3}{|l|}{$\begin{array}{l}\text { Determined by using endoscopy estimates for both high and low confidence } \\
\text { readings of polyps } \leq 5 \mathrm{~mm} \text { and pathology for polyps } \geq 6 \mathrm{~mm}\end{array}$} \\
\hline Colonoscopist & $57[48.2-65.8]$ & $1.49[1.12-1.85]$ \\
\hline Reviewer & $48.8[39.9-57.7]$ & $1.31[0.96-1.67]$ \\
\hline \multicolumn{3}{|l|}{$\begin{array}{l}\text { Determined by using endoscopy estimates for high confidence readings of } \\
\text { polyps }<10 \mathrm{~mm} \text { in size, and pathology for polyps } \geq 10 \mathrm{~mm} \text { in size or for polyps } \\
<10 \mathrm{~mm} \text { in size with low confidence }\end{array}$} \\
\hline Colonoscopist & $55.4[46.5-64.3]$ & $1.41[1.05-1.78]$ \\
\hline Reviewer & $51.2[42.3-60.1]$ & $1.35[0.98-1.71]$ \\
\hline \multicolumn{3}{|l|}{$\begin{array}{l}\text { Determined by using endoscopy estimates for both high and low confidence } \\
\text { readings of polyps }<10 \mathrm{~mm} \text { in size, pathology for polyps } \geq 10 \mathrm{~mm} \text { in size }\end{array}$} \\
\hline Colonoscopist & $56.2[47.4-65]$ & $1.46[1.1-1.83]$ \\
\hline Reviewer & $51.2[42.3-60.1]$ & $1.36[0.99-1.72]$ \\
\hline
\end{tabular}

Table 4 Adenoma detection rate and adenomas per colonoscopy using all endoscopy estimates irrespective of pathology reading.

ADR, adenoma detection rate; APC, adenomas per colonoscopy.

APC, respectively, compared with the ADR and APC determined by pathologic assessment of all polyps.

- Table 4 shows how photography would represent ADR and APC in a post-credentialing phase, as lesions that are false-positive adenoma interpretations would still be counted toward ADR and APC. Under these circumstances, the ADR determined by the reviewer was $48.8 \%$ (absolute reduction of $3.3 \%$ from the pathology-based ADR) and the APC was 1.31 , which is almost identical to the APC determined by pathology ( $\bullet$ Table 4 ).

\section{Discussion}

$\nabla$

This study prospectively evaluated whether an expert colonoscopist could utilize photography during colonoscopy to establish adequate rates of adenoma detection. When the colonoscopist's skill at interpreting adenomas was assessed against pathologically proven adenomas, there was about a $13 \%$ relative reduction in ADR and APC (compared with the pathologically determined
ADR and APC; absolute reductions of $6.6 \%$ and 0.17 , respectively), as determined by an experienced reviewer assessing the endoscopic photographs. This suggests that for a colonoscopist to use photography, and establish competence during a validation or credentialing phase, the colonoscopist's "true" ADR as determined by pathology would need to be somewhat higher than the minimum target ADR of $20 \%$ [2]. Assuming a target ADR of $20 \%$ to establish competent mucosal inspection by any colonoscopist, and an approximately $13 \%$ reduction in ADR with photography, colonoscopists with "true ADRs" of $\geq 24 \%$ should be able to utilize photography to demonstrate adequate ADR. In reality, many colonoscopists have true ADRs substantially above current targets, and therefore should be able to easily establish adequate an ADR using photography. Similar arguments can be put forward regarding the use of APC as a target. A recent study found strong correlation between ADR and APC, and suggested that an APC of 0.5 for males and 0.2 for females, corresponds to the current targets for ADR [21]. 
The current study found that when photography was used to demonstrate ADR and APC without reference to pathology, as it would likely be when implemented in clinical practice, the colonoscopist's calculation of ADR and APC sometimes numerically exceeded the pathologically determined ADR and APC, though the differences were minor. It is possible that for some polyps, the colonoscopist's assessment can provide better information than pathology: in some cases polyps may not be retrieved, or very small adenomas may be removed by cold snaring and sent for pathology where they may be inaccurately characterized as "normal" because the polyp was not sectioned through the adenoma tissue in the pathology laboratory [11].

The concept presented in this study has implications for endoscopic electronic report-generating systems and electronic health records. As resect and discard enters clinical practice, colonoscopists and quality improvement programs will demand that reporting systems allow storage of images of the same quality and resolution as the real-time images that led to the original endoscopic estimation of pathology. These images will also be needed to provide medical-legal protection for colonoscopists, which may be needed if patients subsequently develop colorectal cancer.

The colonoscopist and the reviewer in the current study utilized the NICE criteria [18] to classify polyps. The NICE criteria can be used to differentiate conventional adenomas from polyps in the serrated class, but neither the NICE criteria nor any other endoscopic criteria have been reliably shown to differentiate polyps within the serrated class as hyperplastic vs. SSA/Ps. Similarly, the NICE criteria do not allow identification within conventional adenomas of the degree of dysplasia or villous vs. tubular elements. This lack of information about conventional adenomas is managed in the resect and discard paradigm by confining endoscopic estimations to polyps $\leq 5 \mathrm{~mm}$ in size. Polyps of this size have a very low prevalence of villous elements, high grade dysplasia, or cancer [22]. The lack of information about hyperplastic vs. SSA/P histology can be similarly managed by confining endoscopic estimation to lesions $\leq 5 \mathrm{~mm}$ in size, as the prevalence of SSA/P in lesions of this size is very low [23]. Alternatively, colonoscopists could send serrated lesions of $\leq 5 \mathrm{~mm}$ in size in the proximal colon to pathology. Either management strategy can be incorporated into a resect and discard paradigm.

One concern in establishing detection targets is the potential for any target to be manipulated, and this has been observed in clinical practice [6]. In a photography-based system for documentation of ADR or APC, a photograph of each polyp serves as a substitute for a glass slide stored in the pathology department as the record of the polyp [9]. This system would seem to be resistant to manipulation, but developers of image storage systems and managers of quality improvement programs should direct their development of photography-based ADR to systems that cannot be manipulated.

Limitations of the current study include that it was performed by a single expert colonoscopist and reviewed by a single experienced endoscopist, and both the colonoscopist and the reviewer had extensive experience in NBI. However, this proof-of-concept study demonstrates the feasibility and reasonable accuracy of ADR and APC documentation by photography. The calculated ADR and APC were not substantially affected by use of only high confidence vs. high + low confidence readings. The colonoscopist had higher accuracy for determining pathology than the reviewer, perhaps because of greater experience or evaluation in real time rather than by still photographs, or because of the very lim- ited clinical information about each polyp (e.g. location in the colon) provided to the reviewer. This difference is also a strength of the study, as it indicates that a high-level detector can demonstrate adequate ADR and APC when his/her polyp photographs are studied by reviewers with a range of polyp differentiation experience and skills, as might often occur in clinical practice.

A second limitation is that the study population was largely surveillance patients, and ADR is typically higher (about 7\%) in surveillance patients compared with screening patients [21]. However, the colonoscopist in the current study had twice previously demonstrated ADRs in screening patients that were higher than the $52 \%$ ADR demonstrated in the current study $[15,24]$. Furthermore, there is no indication that the surface features of colorectal polyps in screening patients differ from those in surveillance patients.

In conclusion, these findings suggest that under a wide range of assumptions, high-level detectors should have little difficulty documenting adequate ADR and APC using photography of colorectal polyps. Difficulty in documentation of ADR is an argument against a resect and discard policy. Although many studies have evaluated NBI [25] and other technologies [25,26], this study is, to our knowledge, the first to directly address whether photography can support ADR and APC documentation. ADR and APC determination using photography alone is adequate compared with the pathology-based gold standard. Additional study by other colonoscopists in different practice settings is warranted.

Competing interests: Dr. Rex has received research support from and is a consultant for Olympus.

\section{Acknowledgments \\ $\nabla$}

This work was funded by a gift from Scott and Kay Schurz of Bloomington, Indiana.

\section{References}

1 Rex DK, Bond JH, Winawer S et al. Quality in the technical performance of colonoscopy and the continuous quality improvement process for colonoscopy: recommendations of the U.S. Multi-Society Task Force on Colorectal Cancer. Am J Gastroenterol 2002; 97: 1296-308

2 Rex DK, Petrini JL, Baron TH et al. Quality indicators for colonoscopy. Gastrointest Endosc 2006; 63: 16-28

3 Kaminski MF, Regula J, Kraszewska E et al. Quality indicators for colonoscopy and the risk of interval cancer. N Engl J Med 2010; 362: $1795-1803$

4 Corley $D$, Jensen $C D$, Marks AR et al. Adenoma detection rate and risk of colorectal cancer and death. N Engl J Med 2014; 370: 1298-1306

5 Rex DK, Hewett DG, Snover DC. Detection targets for colonoscopy: from variable detection to validation. Am J Gastroenterol 2010; 105: 2665 2669

6 Wang HS, Pisegna J, Modi $R$ et al. Adenoma detection rate is necessary but insufficient for distinguishing high versus low endoscopist performance. Gastrointest Endosc 2013; 77: 71 - 78

7 Kahi CJ, Ballard D, Shah AS et al. Impact of a quarterly report card on colonoscopy quality measures. Gastrointest Endosc 2013; 77: 925 931

8 Rex DK. Update on colonoscopic imaging and projections for the future. Clin Gastroenterol Hepatol 2010; 8: 318-321

9 Rex DK, Kahi C, O'Brien $M$ et al. The American Society for Gastrointestinal Endoscopy PIVI (Preservation and Incorporation of Valuable Endoscopic Innovations) on real-time endoscopic assessment of the histology of diminutive colorectal polyps. Gastrointest Endosc 2011; 73: 419-422

10 Kaminski MF, Hassan C, Bisschops $R$ et al. Advanced imaging for detection and differentiation of colorectal neoplasia: European Society of 
Gastrointestinal Endoscopy (ESGE) Guideline. Endoscopy 2014; 46: 435- 449

11 Rex DK. Narrow-band imaging without optical magnification for histologic analysis of colorectal polyps. Gastroenterology 2009; 136: $1174-$ 1181

12 Ignjatovic A, East J, Suzuki $N$ et al. Optical diagnosis of small colorectal polyps at routine colonoscopy (Detect InSpect ChAracterise Resect and Discard; DISCARD trial): a prospective cohort study. Lancet Oncol 2009; 10: $1171-1178$

13 Kessler WR, Klein RW, Wielage RC et al. Cost savings of removing diminutive polyps without histologic assessment. Gastrointest Endosc 2008; 67: AB105

14 Hassan C, Pickhardt PJ, Rex DK. A resect and discard strategy would improve cost-effectiveness of colorectal cancer screening. Clin Gastroenterol Hepatol 2010; 8: 865 - 869

15 Rex DK, Helbig CC. High yields of small and flat adenomas with highdefinition colonoscopes using either white light or narrow band imaging. Gastroenterology 2007; 133: $42-47$

16 Kahi Cl, Hewett DG, Norton DL et al. Prevalence and variable detection of proximal colon serrated polyps during screening colonoscopy. Clin Gastroenterol Hepatol 2011; 9: 42 -46

17 Hewett DG, Huffman ME, Rex DK. Leaving distal colorectal hyperplastic polyps in place can be achieved with high accuracy by using narrowband imaging: an observational study. Gastrointest Endosc 2012; 76: $374-380$

18 Hewett DG, Kaltenbach T, Sano Y et al. Validation of a simple classification system for endoscopic diagnosis of small colorectal polyps using narrow-band imaging. Gastroenterology 2012; 143: 599-607
19 Raghavendra $M$, Hewett $D G$, Rex $D K$. Differentiating adenomas from hyperplastic colorectal polyps: narrow-band imaging can be learned in 20 minutes. Gastrointest Endosc 2010; 72: 572 - 576

20 Do A, Weinberg J, Kakkar A et al. Reliability of adenoma detection rate is based on procedural volume. Gastrointest Endosc 2013; 77: 376-380

21 Kahi CJ, Vemulapalli KC, Johnson CS et al. Improving measurement of the adenoma detection rate and adenoma per colonoscopy quality metric: the Indiana University experience. Gastrointest Endosc 2014; 79: $448-454$

22 Lasisi F, Mouchli A, Riddell R et al. Agreement in interpreting villous elements and dysplasia in adenomas less than one centimetre in size. Dig Liver Dis 2013; 45: 1049-1055

23 Abdeljawad K, Vemulapalli KC, Kahi CJ et al. Sessile serrated polyp prevalence determined by a colonoscopist with a high lesion detection rate and an experienced pathologist. Gastrointest Endosc In press 2014: DOI 10.1016/j.gie.2014.04.064

24 Kahi CJ, Anderson JC, Waxman I et al. High-definition chromocolonoscopy vs. high-definition white light colonoscopy for average-risk colorectal cancer screening. Am J Gastroenterol 2010; 105: 1301 - 1307

25 McGill SK, Evangelou E, Ioannidis JP et al. Narrow band imaging to differentiate neoplastic and non-neoplastic colorectal polyps in real time: a meta-analysis of diagnostic operating characteristics. Gut 2013; 62: $1704-1713$

26 Wanders LK, East JE, Uitentuis SE et al. Diagnostic performance of narrowed spectrum endoscopy, autofluorescence imaging, and confocal laser endomicroscopy for optical diagnosis of colonic polyps: a metaanalysis. Lancet Oncol 2013; 14: 1337-1347 